\title{
Guias alimentares para crianças: aspectos históricos e evolução
}

\author{
Food guides for children: historical aspects \\ and evolution
}

Roseane Moreira Sampaio BARBOSA ${ }^{1,2}$

Rosana SALLES-COSTA ${ }^{3}$

Eliane de Abreu SOARES 3,4

\section{RE S U M O}

Os guias alimentares possuem duas propostas: a primeira, ser um guia de saúde pública, e a segunda, uma ferramenta de educação nutricional. Este trabalho objetiva apresentar um histórico dos guias alimentares e suas características, bem como abordar os guias dietéticos desenvolvidos especificamente para crianças. Foi realizado um levantamento bibliográfico dos últimos dez anos, em base de dados Medline utilizando as palavras-chave guia alimentar, guia dietético infantil e pirâmide alimentar infantil. Desde 1916, os guias alimentares vêm sendo desenvolvidos a fim de traduzir as recomendações de uma dieta saudável para população, porém somente a partir de 1999 foi desenvolvido pelo United States Departament of Agriculture um guia alimentar para crianças. Observou-se que os guias alimentares infantis, de modo geral, foram elaborados recentemente. Poucos países os desenvolveram de acordo com o hábito alimentar das crianças, utilizando alimentos típicos da idade e tamanho das porções específicas para essa faixa etária, considerando a limitada capacidade gástrica das crianças. Conclui-se que é muito importante desenvolver, implementar e validar os guias alimentares infantis, pois são uma ferramenta de educação nutricional para a formação de hábitos saudáveis e para a prevenção de doenças crônicas.

Termos de indexação: criança; educação nutricional; guias alimentares; pirâmide alimentar.

A B S T R A C T

Food guide have two proposals: the first, as a public health guide and the second, as a nutritional educational tool. This study aimed at presenting a history of food guides and their characteristics, as well as approaching the dietary guides specifically developed for children. A bibliographical research of the last ten years was

\footnotetext{
1 Mestranda em Nutrição, Universidade Federal do Rio de Janeiro. Rio de Janeiro, RJ, Brasil.

2 Fundação Ataulpho de Paiva. Rio de Janeiro. RJ, Brasil.

3 Instituto de Nutrição Josué de Castro, Centro de Ciências da Saúde, Universidade Federal do Rio de Janeiro. Rio de Janeiro, RJ, Brasil.

4 Departamento de Nutrição Básica e Experimental, Instituto de Nutrição, Universidade do Estado do Rio de Janeiro. R. São Francisco Xavier, 524, $12^{\circ}$ andar, Bloco D, Maracanã, 20550-900, Rio de Janeiro, RJ, Brasil. Correspondência para/Correspondence to: E.A. SOARES.
} 
carried out in the Medline database, using the key words: food guide, dietary guidelines for children and food guide pyramid for young children. Food guides have been developed since 1916, translating the recommendations for a healthy diet to the population, but only in 1999 did the United States Department of Agriculture develop a food guide for children. It was observed that, in general, the food guides for young children were elaborated recently. Few countries have developed them according to the food habits of children, using foods typical of their age range and appropriate portion sizes, considering the limited gastric capacity of children. It was concluded that it was very important to develop, implement and validate food guides for young children, since they are a nutritional educational tool for the formation of healthy habits and the prevention of chronic diseases.

Indexing terms: child; nutrition education; food guides; food guide pyramid.

\section{N T R O D U Ç Ã O}

Passadas três décadas, observa-se que o estado nutricional das crianças americanas vem melhorando. Esse fato é verificado pela diminuição das taxas de mortalidade infantil e pelo declínio das deficiências nutricionais. Entretanto, o número de crianças com sobrepeso vem aumentando, desde 1970, incluindo a população de baixa renda. Aproximadamente, $10,4 \%$ das crianças entre dois e cinco anos de idade e 15,3\% daquelas de seis a onze anos estão com sobrepeso'.

No Brasil, igualmente, tal transição nutricional vem ocorrendo em conseqüência de mudanças seculares nos padrões nutricionais, associadas à modificação da estrutura alimentar, demográfica, socioeconômica, epidemiológica e de estilo de vida das populações ${ }^{2}$. Segundo Monteiro et al. ${ }^{3}$, essa transição é comumente caracterizada pela diminuição da desnutrição e pelo aumento da obesidade. Mudanças no padrão do consumo alimentar foram avaliadas em estudo realizado com a população da América Latina, tendo sido verificado aumento na ingestão total de gorduras, carnes e açúcares e diminuição de cereais, frutas e vegetais ${ }^{4}$.

Como conseqüência, os guias dietéticos para crianças têm se desenvolvido tendo como foco tanto a desnutrição e deficiências nutricionais, quanto o sobrepeso em crianças ${ }^{1}$. Esses guias têm sido utilizados para descrever as recomendações quantitativas e qualitativas dos padrões dietéticos, baseados em evidências científicas 5 . De acordo com a Organização Mundial de Saúde (1996)', "os guias alimentares oferecem recomendações dietéticas, através de comunicados à população, para promover o bem-estar nutricional "6; podendo ser expressos sob a forma de orientações nutricionais, ou através de grupos alimentares ${ }^{5}$.

A principal chave para desenvolver um guia alimentar é a identificação dos problemas de saúde pública mais relevantes na população estudada, e a determinação de quais deles estão relacionados com a dieta. Em 1995, a Food and Agriculture Organization (FAO) e a World Health Organization (WHO) realizaram uma conferência articulada, traçando diretrizes para o desenvolvimento dos guias alimentares. Esse boletim técnico foi divulgado nos congressos, permitindo que qualquer país ou região pudesse iniciar o desenvolvimento de seu guia alimentar específico. O conteúdo desse boletim reforça que o principal fator para desenvolver um guia alimentar é a identificação de problemas relacionados com a dieta ${ }^{7}$. Os guias alimentares podem ser representados por expressão gráfica. Alguns países, como, por exemplo, os Estados Unidos, México, Panamá e Chile, optaram pelo formato da pirâmide, o Canadá escolheu a forma de um arco-íris, a Costa-Rica o formato de uma pizza e a Guatemala optou pela representação gráfica de um pote de cerâmica. Outros países, como Cuba e Venezuela, não utilizam representação gráfica. O número de mensagens nos guias oscila entre seis e oito, evitando um grande número de informações a fim de que a população possa assimilar com facilidade as orientações alimentares $^{8}$.

Portanto, este trabalho foi realizado com o objetivo de apresentar um histórico dos guias 
alimentares e suas características, bem como fazer uma revisão dos guias dietéticos desenvolvidos especificamente para crianças. Destaca-se a importância de abordar esse tema devido à escassez de publicações e à importância de guias infantis para promoção de hábitos saudáveis e prevenção de doenças crônicas.

Foi realizado um levantamento bibliográfico dos últimos dez anos em banco de dados Medline, utilizando como palavras-chave "guias alimentares", "pirâmide alimentar infantil" e "crianças".

\section{Guias alimentares}

Há duas décadas, o Departamento de Serviço Humano e Saúde (Departament of Health and Human Service - HHS) e o Departamento de Agricultura dos Estados Unidos (United States Departament of Agriculture - USDA) vêm publicando os guias alimentares ${ }^{9}$.

O papel da alimentação e da nutrição na promoção da saúde e prevenção de doenças iniciou-se no final de 1800, com a teoria dos microorganismos ${ }^{10}$. Segundo essa teoria, o aparecimento das doenças estava relacionado com a contaminação dos alimentos e da água. Em 1900, Casimiri Funk propôs a teoria da vitamina, segundo a qual o aparecimento de doenças estava relacionado ao consumo inadequado de nutrientes ${ }^{10}$.

O primeiro guia alimentar foi proposto por Caroline Hunt (1916) e traduziu as recomendações de uma dieta saudável. Outros guias alimentares foram publicados, enfocando os problemas de cada época, como a seleção de alimentos durante a Recessão Econômica e alternativas alimentares durante a Segunda Guerra Mundial. Por volta de 1940, após as novas recomendações do National Research Council, de 1941, surgiu o guia alimentar popularmente chamado Basic Seven Food Guide, desenvolvido no período da Segunda Guerra Mundial, com o objetivo de sugerir substituições alimentares em caso de escassez de alimentos, e recomendar alimentos econômicos durante a
Recessão, porém era muito complexo e ainda faltavam as respectivas porções dos alimentos ${ }^{11}$.

No final de 1940, dois estudos, o Twin Cities e o Framingham, começaram a examinar a associação entre fatores dietéticos e o aumento do risco de doenças crônicas não transmissíveis ${ }^{10}$. Por volta de 1950, reconheceu-se que a saúde não é simplesmente ausência de doença e, para garantir o bem-estar, eram necessárias escolhas dietéticas corretas e estilo de vida saudável para a promoção da saúde ${ }^{12}$. Em 1956, especialistas em nutrição publicaram um novo guia alimentar, o Basic Four, com o número de porções recomendadas dos quatro grupos de alimentos, sendo eles: leite, carnes, vegetais e frutas e pães e cereais ${ }^{13}$. A partir da década de 1970, pesquisadores associaram o alto consumo de certos alimentos com o aparecimento de determinadas doenças. Em 1979, foi publicado o Hassle Free Guide, no qual foi incluído o quinto grupo alimentar (gorduras, açúcares, álcool), chamando atenção para o consumo moderado desses alimentos ${ }^{11}$.

Em 1980, após estudo realizado pela Sociedade Americana de Nutrição Clínica, que verificou uma forte associação entre dieta e saúde, o USDA e o HHS publicaram a primeira edição do Guia Alimentar para Americanos, tendo como objetivo o enfoque na saúde, com base no padrão dietético e em dados de consumo e composição dos alimentos, bem como na utilidade para o consumidor. Desde então, esses dois departamentos vêm realizando revisões a cada cinco $\operatorname{anos}^{14}$

Num intervalo de 20 anos, houve uma evolução dos guias alimentares, apresentando muitas diferenças e similaridades. Ao comparar os guias alimentares de 1980 e de 2000 verificou-se que, apesar de as diretrizes serem similares, o número delas aumentou de sete para dez. Observou-se que o guia alimentar de 2000 ficou mais organizado e mudou-se o enfoque das diretrizes aos alimentos: ao invés de ressaltar os alimentos que deveriam ser evitados, optou-se por estimular escolhas alimentares mais saudáveis ${ }^{10}$. 
No guia alimentar de 1980 as sete recomendações eram: (1) consumir uma variedade de alimentos, (2) manter o peso saudável, (3) evitar gordura, gordura saturada e colesterol, (4) ingerir alimentos ricos em carboidratos e fibras, (5) evitar o alto consumo de açúcar, (6) evitar alto consumo de sódio e (7) utilizar bebidas alcoólicas com moderação. Atualmente, no guia de 2000, as dez recomendações são: (1) manter o peso saudável, (2) manter-se fisicamente ativo diariamente, (3) deixar a pirâmide alimentar escolher os alimentos, (4) escolher uma variedade de cereais diariamente, principalmente cereais integrais, (5) escolher uma variedade de frutas e verduras diariamente, (6) guardar os alimentos de forma segura, (7) escolher uma dieta com baixo teor de gordura saturada e colesterol e moderado em gordura total, (8) escolher bebidas e alimentos com moderada quantidade de açúcar, (9) escolher e preparar alimentos com pouca quantidade de sal e (10) consumir álcool moderadamente ${ }^{10}$.

Com relação aos nutrientes, observou-se, igualmente, uma evolução. Em 1980 e 1985, esses guias enfocaram o consumo de carboidratos complexos e fibras. Já em 1990 e 1995, o comitê direcionou o seu enfoque para o consumo de alimentos ricos em fibras e carboidratos. Já em 2000, foi desenvolvido um guia alimentar com diferentes grupos para frutas, vegetais e grãos, pois esses alimentos contêm diferentes nutrientes importantes para a manutenção da saúde ${ }^{15,16}$.

Outro exemplo pode ser visto na recomendação das gorduras. As versões de 1980 e 1985 não especificavam a quantidade de gordura, porém, em 1990, o comitê recomendou que 30\% ou menos do valor energético total (VET) poderia ser oriundo das gorduras totais na dieta e $10 \%$ de gordura saturada. Nas versões mais recentes (1995 e 2000), apresenta-se uma "dieta com baixo teor de gordura, principalmente gordura saturada e colesterol"16.

Desde 1995, a expressão "consumir uma variedade de alimentos" foi incluída na pirâmide alimentar para ilustrar a necessidade dessa variedade, sendo recomendada, pela primeira vez, a prática de atividade física para a manutenção do peso corporal. Em 2000, foi dada mais ênfase na expressão "deixe a pirâmide guiar suas escolhas alimentares" do que na expressão "variedade de alimentos" e uma maior preocupação para a prática de atividade física e manutenção do peso saudável, devido ao crescimento da obesidade ${ }^{10}$.

A mais atual edição dos Guias Alimentares para Americanos foi desenvolvida em 2000. Esse guia fornece um aconselhamento mais específico com relação às escolhas alimentares do que os anteriores. As dez orientações dos guias foram agrupadas em três seções: a primeira é a condição física, que enfatiza os benefícios da prática regular de atividade física; a segunda, a construção de uma base saudável, enfatizando a pirâmide alimentar como base para um padrão alimentar adequado e a escolha de uma variedade de cereais, frutas, vegetais; e a última, as escolhas sensíveis, mostrando os alimentos que devem ser consumidos com moderação, incluindo açúcar, gordura, sal e bebidas alcoólicas ${ }^{9}$.

Com o desenvolvimento das novas recomendações dietéticas do Dietary Reference Intake (DRI) para 26 nutrientes, que foram publicadas a partir de 1997, há necessidade de rever os guias alimentares para garantir o bem-estar e promover a saúde dos indivíduos. Para 2005 está prevista a divulgação do novo guia alimentar para a população americana ${ }^{17}$.

\section{América Latina}

É recente a discussão sobre a presença de guias alimentares nos países da América Latina e a necessidade de desenvolvê-los e implementá-los. Os estudos tiveram início em 1992, com a Conferência Mundial de Nutrição, em Roma, que recomendou a elaboração de guias alimentares de acordo com a realidade e costumes de cada país, no intuito de melhorar os padrões de consumo e o estado nutricional da população ${ }^{8}$. De acordo com Calderón ${ }^{18}$, a situação da elaboração dos guias alimentares, em países da América Latina, 
divide-se em três estágios: guias já elaborados e publicados, guias em fase de elaboração e guias que ainda serão desenvolvidos.

A Venezuela e o Chile já elaboraram e publicaram alguns de seus guias alimentares. No Chile, Yañez et al. ${ }^{19}$ validaram esses instrumentos para crianças em idade escolar, com a finalidade de facilitar o uso da Pirâmide Alimentar. Na Venezuela, foram publicados um guia escolar e outro específico para a população rural ${ }^{18}$.

No Brasil, Plilippi et al. ${ }^{20}$ realizaram uma adaptação da pirâmide alimentar americana para adultos e em 2003 elaboraram, também, para crianças ${ }^{21}$. Em 2002, a Organização Pan-Americana de Saúde e o Ministério da Saúde (OPAS/MS)22 desenvolveram o Guia Alimentar para Crianças de 6 a 23 meses. De acordo com a Coordenação-Geral da Política de Alimentação e Nutrição (CGPNA) do Ministério da Saúde, a elaboração de um Guia Alimentar para População Brasileira está em fase final, e estabelece diretrizes alimentares para o Brasil. O guia divulgará orientações e recomendações para a promoção de estilos de vida saudável, destacando a alimentação, a atividade física e o aleitamento materno. Será direcionado não só ao governo como também à indústria de alimentos e aos profissionais de saúde, com vistas à unificação dos procedimentos.

Os países que se encontram em fase de elaboração dos guias dietéticos para a população são a Venezuela, Argentina, Colômbia e o Equador. Na Venezuela, os guias alimentares estão sendo desenvolvidos para pré-escolares e para alunos da educação básica - de $7^{\mathrm{a}}$ a $9^{\mathrm{a}}$ séries -, embora já tenham sido publicados os guias dietéticos para crianças de $1^{\mathrm{a}}$ a $7^{\mathrm{a}}$ séries $^{18}$. Na Argentina, no ano de 2000, foram desenvolvidos guias alimentares para a população adulta saudável e recomendou-se a elaboração de guias específicos para menores de dois anos ${ }^{18}$. Na Colômbia, estão sendo elaborados os guias para pré-escolares a partir de dois anos, escolares, adolescentes, adultos e idosos ${ }^{18}$. Alguns países, como a Bolívia, Paraguai, Peru e Uruguai, estão iniciando a elaboração desses guias alimentares ${ }^{18}$.
A maioria dos países mencionados desenvolveu guias alimentares para a população adulta e saudável ${ }^{8}$. A inexistência de guias específicos para a população infantil se deve à dificuldade nas adaptações dos conteúdos das mensagens e das porções expressas de alimentos, com o propósito de torná-los compreensíveis e motivadores.

\section{Guias dietéticos para crianças}

Em 1995, a Venezuela publicou "Os guias da alimentação na escola", sendo o volume 1 dirigido para crianças de $1^{\mathrm{a}}$ a $3^{\mathrm{a}}$ série e o volume 2 destinado àquelas de $4^{\mathrm{a}}$ a $7^{\mathrm{a}}$ série. Em ambos, consideraram-se as mesmas mensagens já publicadas, variando conforme a idade e os objetivos específicos de cada programa ${ }^{23}$.

Em 1999, a USDA realizou uma adaptação da pirâmide alimentar para crianças de dois a seis anos de idade, com o objetivo de focar as preferências alimentares e as recomendações nutricionais para essa faixa etária ${ }^{13}$. Essa pirâmide divide os alimentos em cinco grupos: cereais (seis porções), vegetais (três porções), frutas (duas porções), leite (duas porções) e carne (duas porções) e, no topo da pirâmide, estão os grupos das gorduras e dos açúcares, os quais devem ser utilizados com moderação, pois possuem muitas calorias e baixa quantidade de vitaminas e minerais. Os autores ressaltam que, apesar de as gorduras serem necessárias para o crescimento e desenvolvimento de pré-escolares, elas não devem ultrapassar 30\% das calorias totais diárias.

Para as crianças na faixa etária de dois a três anos, o número de porções sugeridas para cada grupo alimentar é o mesmo daquele recomendado para as crianças de dois a seis anos, porém deve equivaler a dois terços do tamanho da porção de cada alimento, exceto para o grupo do leite, cujo porcionamento deve ser o mesmo. Pela primeira vez, na representação gráfica da pirâmide alimentar, foram incluídas crianças realizando algumas atividades físicas (jogando bola, pulando corda, correndo, brincando), 
ressaltando a importância da prática de atividade física regular nessa faixa etária. Além disso, são apresentadas algumas orientações quanto à forma em que as preparações devem ser oferecidas às crianças. Os alimentos podem se tornar perigosos, pois há a possibilidade de a criança engasgar, portanto, deve-se fazer a escolha por lanches saudáveis e enfatizar a importância de oferecer, diariamente, uma variedade de alimentos ${ }^{13}$.

A Austrália, em 2003, realizou uma revisão do seu guia dietético para crianças e adolescentes, desenvolvido, em 1995, pelo National Health and Medical Research Council (NH\&MRC). Esse guia alimentar é apresentado sob a forma de orientações que recomendam a amamentação, o monitoramento do crescimento, a prática de atividade física, a escolha de alimentos de vários grupos (vegetais, frutas, carnes, leite e cereais), incentivam o consumo de água e chamam atenção para a não utilização de bebidas alcoólicas pelas crianças; com relação às gorduras, orientam a limitação das gorduras saturadas e totais e a escolha de alimentos com pouco sal, bem como o consumo moderado de açúcar e açúcar de adição ${ }^{24}$.

No Canadá, o guia alimentar para pré-escolares foi desenvolvido, em 1995, com base no guia alimentar para adultos, pois as crianças têm de se adaptar à alimentação do adulto. Esse guia alimentar tem o formato de um arco-íris e é dividido em quatro grupos alimentares: cereais (5-12 porções), vegetais e frutas (5-10 porções), leite (2-3 porções), carnes (2-3 porções). Existe uma ampla variação nas porções, visando atender toda a família; para o pré-escolar, recomenda-se a menor porção, além disso, o tamanho da porção dos alimentos também é menor, por exemplo, no grupo dos cereais, uma porção de pão para crianças é meia fatia, em vez de uma fatia. Esse guia chama atenção para as características do pré-escolar, que está em uma fase de intenso aprendizado, imitação e independência, sendo a criança capaz de aceitar um alimento e, dias depois, rejeitá-lo. Indica-se o consumo de uma variedade de alimentos a fim de garantir a recomendação nutricional e o contato com diversas texturas, sabores, cores e aromas. Devem-se consumir carboidratos complexos e gorduras moderadamente, mas tendo sempre o objetivo de atender à quantidade de energia necessária ao crescimento e desenvolvimento do pré-escolar ${ }^{25}$.

Várias instituições têm estabelecido guias de consumo alimentar para melhor orientar a população na escolha de alimentos saudáveis e porções adequadas. Em 1999, USDA desenvolveu a pirâmide alimentar para criança ${ }^{13}$. Essa pirâmide é conhecida como uma ferramenta de educação nutricional e recomenda que a dieta contenha o número de porções adequado dos cinco grupos de alimentos ${ }^{26}$.

No Brasil, apenas dois guias alimentares para crianças foram desenvolvidos. Em 2002, uma iniciativa conjunta da Organização Pan-Americana de Saúde (OPAS) com o Ministério da Saúde (MS) ${ }^{22}$ desenvolveu o Guia Alimentar para Crianças de 6 a 23 meses, propondo, além da pirâmide alimentar para essa faixa etária, dez passos para uma alimentação saudável. Esse guia é uma ferramenta prática que permite aos responsáveis a seleção de uma alimentação saudável, incluindo informações sobre higiene dos alimentos, estímulo à amamentação e introdução dos alimentos após o desmame. Enfatiza a variedade e a consistência adequada dos alimentos. Os alimentos selecionados devem ser do hábito alimentar da família, ajustados em quantidade e qualidade. A pirâmide alimentar é constituída por oito grupos de alimentos, distribuídos em quatro níveis, apresentados da base ao topo da pirâmide, considerando a sua participação na dieta em quantidades, respectivamente das maiores às menores, quanto ao número de porções. A organização da pirâmide é feita em função do principal nutriente de cada tipo de alimento e a quantidade necessária. Um alimento não é mais importante que outro por sua localização, todos os grupos são importantes e têm suas funções. Os grupos são assim formados: grupo (1): cereais, pães e tubérculos (3-5 porções); grupo (2): verduras e legumes (3 porções); grupo (3): frutas (3-4 porções); grupo (4): leites, queijos e iogurtes (3 porções); grupo (5): carnes e ovos (2 porções); grupo (6): leguminosas (1 porção); grupo (7): óleos e gorduras (2 porções); grupo (8): açúcares e doces (1 porção)22 (Figura 1). 


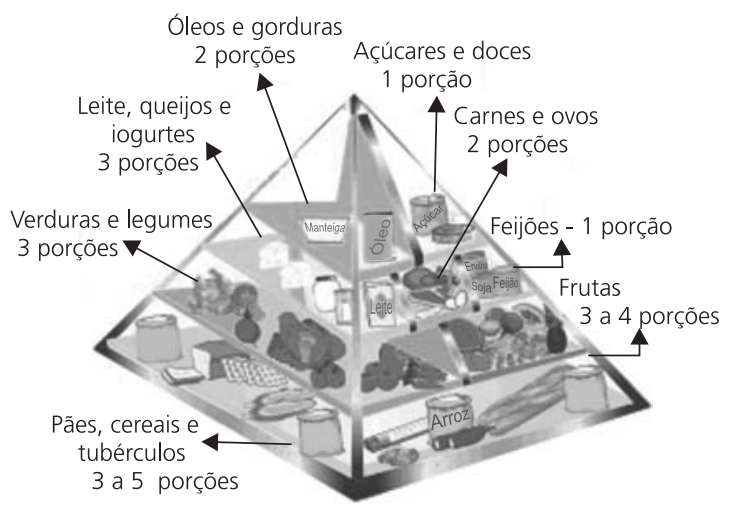

Figura 1. Pirâmide alimentar infantil de crianças de 6 a 23 meses. Fonte: OPAS ${ }^{22}$.

O outro guia brasileiro foi desenvolvido por Philippi et al. ${ }^{21}$ e é um instrumento para orientação nutricional baseado na proposta da pirâmide alimentar norte-americana, adaptada às crianças brasileiras de dois e três anos de idade. Essa pirâmide foi baseada em uma dieta padrão, planejada para essa faixa etária, contendo os alimentos mais comumente consumidos. Também é dividida em oito grupos: arroz, pão, massa, batata, mandioca (cinco porções), verduras e legumes (três porções), frutas (três porções), carnes e ovos (duas porções), leite, queijo e iogurte (três porções), leguminosas (uma porção), óleos e gorduras (uma porção) e açúcares e doces (uma porção). Além disso, é recomendado escolher uma dieta variada com alimentos de todos os grupos da pirâmide, dar preferência aos vegetais, como frutas, verduras e legumes, ficar atento ao modo de preparo dos alimentos, procurando facilitar a mastigação e deglutição pelas crianças e dando prioridade aos alimentos em sua forma natural e às preparações assadas, cozidas em água ou vapor e grelhadas. Os autores ressaltam, também, as preparações culinárias, que devem ser elaboradas de modo a atrair a atenção das crianças, orientam a leitura dos rótulos dos alimentos infantis industrializados para conhecer o valor nutritivo do alimento e seu modo de preparo. Os autores afirmam que: a introdução de novos alimentos e preparações deve ser feita de forma gradual e freqüente para que a criança possa aprovar e incluir em seus hábitos alimentares; açúcares, doces, sal e alimentos ricos em sódio devem ser utilizados com moderação; devem-se consumir alimentos com baixo teor de gordura total, dando preferência às carnes magras; devem-se usar gorduras poliinsaturadas encontradas em óleos vegetais (girassol, milho, canola e soja) ${ }^{21}$ (Figura 2).

\section{Diferenças e limitações dos guias alimentares infantis}

Uma grande limitação dos guias alimentares se refere ao grupo das gorduras e dos açúcares, uma vez que a recomendação se dá por meio de expressões como: "uso moderado", "pequena quantidade", "quantidade razoável", dificultando sua interpretação. Nos guias alimentares infantis desenvolvidos no Brasil, recomenda-se a quantidade que deve ser ingerida para esses dois grupos. A Organização Pan-Americana de Saúde ${ }^{22}$ e Philippi et al. ${ }^{21}$ recomendam uma porção do grupo das gorduras e uma porção do grupo dos açúcares para crianças.

Outra dificuldade é que muitos alimentos que são ingeridos como preparação devem ser

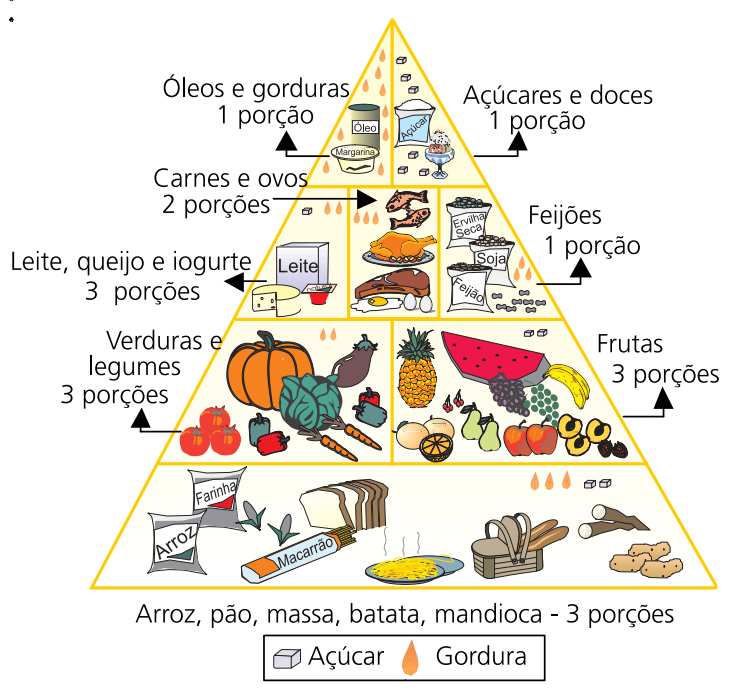

Figura 2. Pirâmide alimentar brasileira infantil - 2 a 3 anos de idade.

Fonte: Philippi et al. ${ }^{21}$. 
classificados em mais de um grupo de alimentos, necessitando que os ingredientes sejam desmembrados e, por conseguinte, que se tenha conhecimento dos ingredientes que compõem a preparação.

Tanto nos guias desenvolvidos nos Estados Unidos como no Canadá, foram utilizados os mesmos alimentos recomendados para adultos, nos diversos grupos alimentares, alterando somente o tamanho da porção do alimento oferecido às crianças. No Brasil, os guias infantis foram elaborados com alimentos e quantidades característicos dessa faixa etária, considerando a limitada capacidade gástrica das crianças e os alimentos comumente consumidos.

Nota-se uma diferença em relação à divisão dos grupos alimentares dos países citados. A USDA e a Austrália recomendam, na pirâmide alimentar, cinco grupos, no Canadá recomendam-se quatro grupos e no Brasil, oito grupos. Nota-se que a pirâmide desenvolvida no Brasil é a única que separa o grupo das carnes e das leguminosas, além de incluir os alimentos ricos em amido (batata, aipim, inhame) no grupo dos cereais. 0 grande problema de incluir os tubérculos e raízes no grupo dos vegetais é que se pode superestimar o consumo de vegetais, devido ao alto consumo desses alimentos pelas crianças.

Ressalta-se a importância dada à pirâmide alimentar infantil da USDA com relação à prática da atividade física, sendo representada junto com os grupos alimentares. Já no Brasil, essa orientação não foi ressaltada.

\section{O N CLUSÃ O}

Os guias alimentares representam um importante instrumento para atender os objetivos dos programas de educação nutricional, pois facilitam a aprendizagem e adaptação de uma conduta alimentar saudável. Esses guias devem se ajustar às recomendações de energia e nutrientes de cada país.

Países e regiões que se comprometeram com o processo de elaboração de guias alimentares reconhecem os vários problemas e questões a serem abordados em sua implementação.
Alguns desses desafios incluem a tradução dos guias para várias línguas e dialetos, motivação da mudança do comportamento alimentar dos indivíduos, bem como a sua conscientização e a garantia de que os guias alimentares sejam direcionados a todos os níveis socioeconômicos e de escolaridade da população, mantendo o entendimento das mensagens envolvidas.

Os guias alimentares infantis foram elaborados recentemente, e poucos países os desenvolveram de acordo com o hábito alimentar dessa fase e com características alimentares do país. O grupo de crianças menores de dois anos deve ser objeto de recomendações especiais, devendo considerar fatores econômicos, psicossociais, culturais e de escolaridade da população. $O$ número de recomendações não deve ultrapassar dez, sendo mencionados aspectos relacionados à promoção do estilo de vida saudável, como atividade física e higiene alimentar. As representações gráficas são específicas para cada país e devem expressar os conceitos de variedade, freqüência e proporção dos alimentos.

No Brasil, tanto o guia alimentar para crianças menores de dois anos da Organização Pan-Americano de Saúde ${ }^{22}$ quanto o desenvolvido por Philippi et al. ${ }^{21}$ para crianças de dois e três anos tiveram o tamanho das porções adaptado à faixa etária, considerando a limitada capacidade gástrica das crianças nessa idade e o uso de alimentos típicos dessa faixa etária.

Ressalta-se a importância de desenvolver, implementar e validar os guias alimentares infantis, pois eles são um guia de saúde pública e uma ferramenta de educação nutricional. Além disso, devido a mudanças nos padrões nutricionais ocorridas nos últimos anos, eles têm objetivo de promover hábitos saudáveis e prevenir doenças crônicas não transmissíveis.

\section{REFERÊ NCIAS}

1. American Dietetic Association. Position of the American Dietetic Association: dietary guidance for healthy children ages 2 to 11 years. J Am Diet Assoc. 2004; 104(4):660-77. 
2. Popkin BM. The nutrition transition in low-income countries: an emergin crisis. Presented at the diet and chronic diseases in countries en socio economic transition. Exper Biol Confer. 1994; p.24-8.

3. Monteiro CA, Mondini L, Souza, ALM, Popkin BM. Da desnutrição para a obesidade: a transição nutricional no Brasil. In: Monteiro CA. Velhos e novos males da saúde no Brasil: a evolução do país e de suas doenças. 2.ed. São Paulo: Hucitec; 2000. p.247-55.

4. Bermudez Ol, Tucker KL. Trends in dietary patterns Latin American populations. Cad Saúde Pública. 2003; 19(1):87-99.

5. Harris S, Black R, Harvey AG. Dietary guidelines: past experience and new approaches. Am J Diet Assoc. 2003; 103(Supl 2):S3-S4.

6. World Health Organization. Preparation and use of Food-Based Dietary Guidelines. Nutrition Programme. Geneva; 1996.

7. World Health Organization. Preparation and use of food-based dietary guidelines. Geneva; 1998. Technical Report Series 880.

8. Peña M. Guias de Alimentación en América Latina. In Anais do I Workshop Instituto Danone. Alimentação equilibrada para a população brasileira. Florianópolis: Instituto Danone; 1998. p.31-43.

9. MacMurry KY. Setting dietary guidelines: the US process. J Am Diet Assoc. 2003; 103(12):10-6.

10. Schneeman BO. Evolution of dietary guidelines. J Am Diet Assoc. 2003; 103(12):5-9.

11. Myers EF, Britten P, Davis CA. Past, present, and future of the food guide pyramid. J Am Diet Assoc. 2001; 101(8):881-5.

12. Committee on Health and Behavior: Research, pratice and policy. The interplay of biological, behavioral and societal influences. Washington, DC: National Academy of Sciences; 2001.

13. Davis CA, Escobar A, Marcoe KL, Tarone C, Shaw $A$, Saltos $S$, et al. Food guide pyramid for young children 2 to 6 years old: Technical Report on Background and Development. U.S. Department Agriculture, Center for Nutrition Policy and Promotion; 1999. CNPP-10.

14. Nutrition and your Health: Dietary Guidelines for Americans. Washington, DC: US Department of Agriculture of Health and Human Services; 1980.

15. United States Department of Agriculture and Department of Health and Human Service. Nutrition and your healthy: dietary guidelines for americans, home and garden bulletin 232. Washington (DC): 2S Government Printing Office; 1980.

16. Dietary Guidelines Advisory Committee. 2000 Report. Washington (DC): US Department Agriculture; 2000.

17. Bush M, Kirkpatrick S. Setting dietary guidance: The Canadian experience. J Am Diet Ass. 2003; 103(12):22-7

18. Calderón T, Morón C. La elaboración de guías alimentarias basadas en alimentos en países de América Latina [acesso 15 maio 2004]. Disponible: http://www.fao.org

19. Yañez ER, Olivares SC, Torres LI, Guevara MN. Validación de las guías y la pirámide alimentaria en escolares de $5^{\circ}$ a $8^{\circ}$ básico. Rev Chil Nutr. 2000; 27(3):358-67.

20. Philippi ST, Latterza, AR, Cruz ATR, Ribeiro LC. Pirâmide alimentar adaptada: guia para escolha dos alimentos. Rev Nutr. 1999; 12(1):65-80.

21. Philippi ST, Cruz ATR, Colucci ACA. Pirâmide alimentar para crianças de 2 e 3 anos. Rev Nutr. 2003; 16(1):5-19.

22. Organização Pan-Americana de Saúde. Dez passos para uma alimentação saudável: guia alimentar para crianças menores de 2 anos. Ministério da Saúde; 2002. 45p.

23. Márquez YF, Cuenca E, Nuñez GO. Las guías alimentares: una estrategia venezolana para educar a la comunidad promoviendo patrones de consumo que mejoren su calidad de vida la educación 2000 [acesso 15 maio 2004]. Disponíble http://www.educoas.org/portal/bdigital/contenido/ laeduca/laeduca_134135/articulo7/index.aspx? culture $=$ es $\&$ tabindex $=2 \&$ childindex $=4$

24. Baghust KI. Dietary Guidelines: The development process in Australia and New Zeland. J Am Diet Assoc. 2003; 103(2 Supl 2):S17-S21.

25. Office of Nutrition Police and Promotion [Internet]. Canada's food guide to healthy eating focus on preschoolers - background for educators and communicators [cited 2004 Mai 15]. Available from: http://www.hc-sc.gc.ca/hpfb-dgpsa/onppbppn/food_guide_preschoolers_e.html\#2

26. Brady LM, Lindquist CH, Herd SL, Goran MI. Comparison of children's dietary intake patterns with US dietary guidelines. Br J Nutr. 2000; 84(3):361-7.

Recebido em:16/8/2004

Versão final reapresentada em: 22/4/2005

Aprovado em: 19/5/2005 